\title{
Summability Properties for Multiplication Operators on Banach Function Spaces
}

\author{
O. Delgado and E. A. Sánchez Pérez
}

\begin{abstract}
Consider a couple of Banach function spaces $X$ and $Y$ over the same measure space and the space $X^{Y}$ of multiplication operators from $X$ into $Y$. In this paper we develop the setting for characterizing certain summability properties satisfied by the elements of $X^{Y}$. At this end, using the "generalized Köthe duality" for Banach function spaces, we introduce a new class of norms for spaces consisting of infinite sums of products of the type $x y$ with $x \in X$ and $y \in Y$.
\end{abstract}

Mathematics Subject Classification (2010). Primary 46E30; Secondary 47B38.

Keywords. Banach function spaces, Köthe dual and generalized dual spaces, product spaces, multiplication operators, summability properties.

\section{Introduction}

Let $(\Omega, \Sigma, \mu)$ be a fixed $\sigma$-finite measure space and consider a couple of Banach function spaces $X$ and $Y$ related to $\mu$. In this paper we introduce a technique based on topological products of Banach function spaces for analyzing the summability properties of the multiplication operators from $X$ into $Y$. For the definition of such topologies we use the so called generalized duality for Banach function spaces, which was originally studied by Maligranda and Persson in [9]. The $Y$-dual space of $X$, denoted by $X^{Y}$, is the space of measurable functions $g$ defining a multiplication operator (also denoted by $g$ ) from $X$ into $Y$, that is, $\langle g, x\rangle=g x \in Y$ for all $x \in X$. This notion includes the classical Köthe dual (or associate) space $X^{\prime}=X^{L^{1}}$.

The first author thanks the support by UPV (PAID-06-08 Ref. 3093), MEC (TSGD-08 and D.G.I. \#MTM2006-13000-C03-01) (Spain) and FEDER. The second author thanks the support by UPV (PAID-06-08 Ref. 3093), MEC (D.G.I. \#MTM2006-11690-C02-01) (Spain) and FEDER. 
Given $1 \leq p<\infty$ and $Z$ another Banach function space related to $\mu$, our goal is to characterize when a multiplication operator $g \in X^{Y}$ is what we call $(p, Z)$-summing, that is, when there exists $K>0$ such that for every $x_{1}, \ldots, x_{n} \in X$,

$$
\left(\sum_{i=1}^{n}\left\|g x_{i}\right\|_{Y}^{p}\right)^{1 / p} \leq K \sup _{f \in B_{X} Z}\left(\sum_{i=1}^{n}\left\|f x_{i}\right\|_{Z}^{p}\right)^{1 / p} .
$$

An operator satisfying this kind of inequality is interesting as it transforms sequences which are summable in a certain weak sense into strongly summable sequences.

Some relevant well known geometric and topological properties involving vector norm inequalities for operators can be written as particular examples of this general class of inequalities when $Z$ is chosen adequately. For instance, in the case when $X$ is order continuous, the positive $p$-summing multiplication operators coincide with the $\left(p, L^{1}\right)$-summing ones, or in the case when $X$ is order semi-continuous and $p$-convex with constant 1 , the $p$ concave multiplication operators coincide with the $\left(p, L^{p}\right)$-summing ones (see Section 4).

Inspired in part by the representation theory of operator ideals as dual spaces of topological tensor products (see for instance [5]), we show that the subspace of $X^{Y}$ of all $(p, Z)$-summing multiplication operators can be described as the Köthe dual of a product space with a particular normed topology given by a certain $d_{p, Z}$-norm. Actually, there is an abuse of the

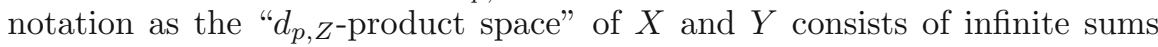
of products of the type $x y$ with $x \in X$ and $y \in Y$. As a consequence of the above description, some factorization theorems for multiplication operators which play a central role in the theory of the Banach function spaces (Reisner and Maurey-Rosenthal's theorems) provide sufficient conditions for $(p, Z)$ summability type properties to hold.

The paper is organized as follows. Section 2 contains the definitions and some results concerning product spaces which will be necessary for our work. In Section 3 we introduce the $d_{p, Z}$-product spaces which will allow us to characterize in Section 4 the $(p, Z)$-summing multiplication operators. Moreover, in Section 4 we show conditions on $X, Y$ and $Z$ guaranteeing that every multiplication operator from $X$ into $Y$ is $(p, Z)$-summing. Examples in which these conditions hold are provided in Section 5 by using the already quoted factorization theorems.

\section{Preliminaries and first results}

Let $(\Omega, \Sigma, \mu)$ be a fixed $\sigma$-finite measure space and denote by $L^{0}$ the space of all (a.e. classes of) real measurable functions defined on $\Omega$. A Banach function space is a Banach space $X \subset L^{0}$ with norm $\|\cdot\|_{X}$, satisfying that if $f \in L^{0}$, $g \in X$ and $|f| \leq|g|$ a.e. then $f \in X$ and $\|f\|_{X} \leq\|g\|_{X}$. Note that in this case, $X$ is a Banach lattice for the pointwise a.e. order. A Banach function 
space is order continuous if every order bounded increasing sequence is norm convergent. A Banach function space $X$ has the Fatou property if for every sequence $\left(f_{n}\right) \subset X$ such that $0 \leq f_{n} \uparrow f$ a.e. and $\sup _{n}\left\|f_{n}\right\|_{X}<\infty$, it follows that $f \in X$ and $\left\|f_{n}\right\|_{X} \uparrow\|f\|_{X}$. A Banach function space $X$ is order semicontinuous if $f, f_{n} \in X$ with $0 \leq f_{n} \uparrow f$ a.e. implies $\left\|f_{n}\right\|_{X} \uparrow\|f\|_{X}$. Of course if a Banach function space $X$ is order continuous or has the Fatou property: then $X$ is order semi-continuous. For issues related to Banach function spaces: see $\left[12\right.$, Ch. 15] considering the function norm $\rho$ defined as $\rho(f)=\|f\|_{X}$ if $f \in X$ and $\rho(f)=\infty$ in other case.

Given two Banach function spaces $X$ and $Y$, the $Y$-dual space of $X$ is defined by

$$
X^{Y}=\left\{h \in L^{0}: h f \in Y \text { for all } f \in X\right\},
$$

that is, the space of functions in $L^{0}$ defining a continuous linear operator from $X$ into $Y$. The continuity follows from the fact that every positive linear operator between Banach lattices is continuous, see [6, p. 2]. The space $X^{Y}$ is a Banach function space with norm

$$
\|h\|_{X^{Y}}=\sup _{f \in B_{X}}\|h f\|_{Y}, \quad \text { for } h \in X^{Y},
$$

if and only if $X$ is saturated, that is, there is no $A \in \Sigma$ with $\mu(A)>0$ such that $f \chi_{A}=0$ a.e. for all $f \in X$, see [9, Proposition 2] and [2, p. 3]. The saturation property is equivalent to the following one: for all $A \in \Sigma$ with $\mu(A)>\mathrm{C}$ there exists $B \in \Sigma$ such that $B \subset A, \mu(B)>0$ and $\chi_{B} \in X$. This is alsc equivalent to $X$ having a weak unit, i.e. a function $g \in X$ such that $g>0$ a.e. As we have already noted, the classical Köthe dual space $X^{\prime}$ coincide with $X^{L^{1}}$, the $L^{1}$-dual space of $X$. In this case, $X^{\prime}$ is saturated whenever $X$ is so. However, the generalized dual $X^{Y}$ of $X$ may be non saturated even if $X$ is saturated. For these and other comments about saturation involving the spaces $X^{Y}$ see [2].

Let us introduce now the product spaces which will be the basic setting

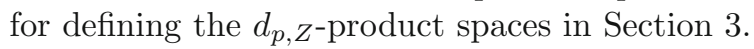

Definition 2.1. The $\pi$-product space $X \pi Y$ is the space of functions $z \in L^{0}$ such that $|z| \leq \sum_{i>1}\left|x_{i} y_{i}\right|$ a.e. for some sequences $\left(x_{i}\right) \subset X$ and $\left(y_{i}\right) \subset Y$ satisfying $\sum_{i \geq 1}\left\|x_{i}\right\|_{X}\left\|y_{i}\right\|_{Y}<\infty$. For $z \in X \pi Y$, consider

$$
\pi(z)=\inf \left\{\sum_{i \geq 1}\left\|x_{i}\right\|_{X}\left\|y_{i}\right\|_{Y}\right\},
$$

where the infimum is taken over all sequences $\left(x_{i}\right) \subset X$ and $\left(y_{i}\right) \subset Y$ such that $|z| \leq \sum_{i \geq 1}\left|x_{i} y_{i}\right|$ a.e. and $\sum_{i \geq 1}\left\|x_{i}\right\|_{X}\left\|y_{i}\right\|_{Y}<\infty$.

The space $X \pi Y$ is clearly an ideal of $L^{0}$ and $\pi(v) \leq \pi(z)$ whenever $|v| \leq|z|$ a.e. It can be routinely checked that $\pi$ is a seminorm. However: there are cases in which $\pi$ is not a norm.

Example. Let $([0,1], \mathcal{B}([0,1]), m)$ be the fixed measure space, where $\mathcal{B}([0,1])$ is the $\sigma$-algebra of all Borel sets of $[0,1]$ and $m$ is the Lebesgue measure on 
$[0,1]$, and consider the product space $L^{1} \pi L^{2}$. For the intervals $A_{i}^{n}=\left[\frac{i-1}{n}, \frac{i}{n}\right]$ with $i=1, \ldots, n$, we have that $\chi_{[0,1]} \leq \sum_{i=1}^{n} \chi_{A_{i}^{n}}$ and

$$
\pi\left(\chi_{[0,1]}\right) \leq \sum_{i=1}^{n}\left\|\chi_{A_{i}^{n}}\right\|_{L^{1}}\left\|\chi_{A_{i}^{n}}\right\|_{L^{2}}=\sum_{i=1}^{n} \frac{1}{n}\left(\frac{1}{n}\right)^{\frac{1}{2}}=\frac{1}{\sqrt{n}} .
$$

Then, taking limit as $n \rightarrow \infty$ we have that $\pi\left(\chi_{[0,1]}\right)=0$, while $\chi_{[0,1]}>0$. So, $\pi$ is not a norm.

Saturation conditions will be crucial for $\pi$ to be a norm under which $X \pi Y$ is a Banach function space. We write " $X \hookrightarrow_{c} Y$ " ( $X \hookrightarrow_{i} Y$ ") if $X$ is continuously contained in $Y$ with $\|x\|_{Y} \leq c\|x\|_{X}\left(\|x\|_{Y}=\|x\|_{X}\right)$ for all $x \in X$. If $X=Y$ with equal norms, we write $X \equiv Y$.

Proposition 2.2. The following conditions are equivalent:

(a) $X \pi Y$ is a saturated Banach function space.

(b) $X, Y$ and $X^{Y^{\prime}}$ are saturated.

Moreover, if (a)-(b) holds, we have that

(i) $X \pi Y \hookrightarrow_{1}\left(X^{Y^{\prime}}\right)^{\prime}$,

(ii) $(X \pi Y)^{\prime} \equiv X^{Y^{\prime}} \equiv Y^{X^{\prime}}$.

Proof. (a) $\Rightarrow$ (b) Let us see that $X$ is saturated. If this is not the case, there exists $A \in \Sigma$ with $\mu(A)>0$ such that $x \chi_{A}=0$ a.e. for all $x \in X$. Since $X \pi Y$ is saturated we can take $B \in \Sigma$ such that $B \subset A, \mu(B)>0$ and $\chi_{B} \in$ $X \pi Y$. Let $\left(x_{i}\right) \subset X$ and $\left(y_{i}\right) \subset Y$ be such that $\sum_{i>1}\left\|x_{i}\right\|_{X}\left\|y_{i}\right\|_{Y}<\infty$ and $\chi_{B} \leq \sum_{i \geq 1}\left|x_{i} y_{i}\right|$ a.e. Then, $\chi_{B}=\chi_{B} \cdot \chi_{A} \leq \sum_{i \geq 1}\left|x_{i} \chi_{A} y_{i}\right|=0$ a.e. and so $\mu(B)=0$, which is a contradiction. Similarly, $Y$ is saturated. Then, the spaces $Y^{\prime}$ and $X^{Y^{\prime}}$ are Banach function spaces. Let us prove that $X^{Y^{\prime}} \equiv(X \pi Y)^{\prime}$ and so we will have that $X^{Y^{\prime}}$ is saturated as $(X \pi Y)^{\prime}$ is so. Let $h \in X^{Y^{\prime}}$. Given $z \in X \pi Y$ and $\left(x_{i}\right) \subset X,\left(y_{i}\right) \subset Y$ with $\sum_{i \geq 1}\left\|x_{i}\right\|_{X}\left\|y_{i}\right\|_{Y}<\infty$ such that $|z| \leq \sum_{i \geq 1}\left|x_{i} y_{i}\right|$ a.e., using the monotone convergence theorem, we have

$$
\int|h z| d \mu \leq \sum_{i \geq 1} \int\left|h x_{i} y_{i}\right| d \mu \leq \sum_{i \geq 1}\left\|h x_{i}\right\|_{Y^{\prime}}\left\|y_{i}\right\|_{Y} \leq\|h\|_{X^{Y^{\prime}}} \sum_{i \geq 1}\left\|x_{i}\right\|_{X}\left\|y_{i}\right\|_{Y}
$$

Then, $\int|h z| d \mu \leq\|h\|_{X_{Y^{\prime}}} \cdot \pi(z)$. So, $h \in(X \pi Y)^{\prime}$ and $\|h\|_{(X \pi Y)^{\prime}} \leq\|h\|_{X^{Y^{\prime}}}$. Consider now $h \in(X \pi Y)^{\prime}$. For every $x \in X$ and $y \in Y$, we have that $x y \in X \pi Y$ and so $h x y \in L^{1}(\mu)$. Then, $h x \in Y^{\prime}$ for every $x \in X$, that is, $h \in X^{Y^{\prime}}$. Moreover, since $\pi(x y) \leq\|x\|_{X}\|y\|_{Y}$ for $x \in X$ and $y \in Y$, it follows

$$
\|h\|_{X^{Y^{\prime}}}=\sup _{x \in B_{X}} \sup _{y \in B_{Y}} \int|h x y| d \mu \leq \sup _{z \in B_{X \pi Y}} \int|h z| d \mu=\|h\|_{(X \pi Y)^{\prime}} .
$$

(b) $\Rightarrow(\mathrm{a})$. Note that from the hypothesis $\left(X^{Y^{\prime}}\right)^{\prime}$ is a Banach function space. Let us see that $X \pi Y \hookrightarrow_{1}\left(X^{Y^{\prime}}\right)^{\prime}$. Given $z \in X \pi Y,\left(x_{i}\right) \subset X,\left(y_{i}\right) \subset Y$ 
such that $\sum_{i \geq 1}\left\|x_{i}\right\|_{X}\left\|y_{i}\right\|_{Y}<\infty$ and $|z| \leq \sum_{i \geq 1}\left|x_{i} y_{i}\right|$ a.e., for every $h \in$ $X^{Y^{\prime}}$,

$$
\int|z h| d \mu \leq \sum_{i \geq 1} \int\left|h x_{i} y_{i}\right| d \mu \leq \sum_{i \geq 1}\left\|h x_{i}\right\|_{Y^{\prime}}\left\|y_{i}\right\|_{Y} \leq\|h\|_{X^{Y^{\prime}}} \sum_{i \geq 1}\left\|x_{i}\right\|_{X}\left\|y_{i}\right\|_{Y}
$$

and so $\int|z h| d \mu \leq\|h\|_{X^{Y^{\prime}}} \cdot \pi(z)$. Then, $z \in\left(X^{Y^{\prime}}\right)^{\prime}$ and $\|z\|_{\left(X^{Y^{\prime}}\right)^{\prime}} \leq \pi(z)$. Hence, $\pi(z)=0$ implies $z=0$ a.e. That is, $\pi$ is a norm on $X \pi Y$.

Let $\left(z_{n}\right)_{n \geq 1} \subset X \pi Y$ be such that $z_{n} \geq 0$ and $\sum_{n \geq 1} \pi\left(z_{n}\right)<\infty$. Let us prove that $\sum_{n \geq 1} z_{n} \in X \pi Y$ (i.e. $X \pi Y$ has the Riesz-Fischer property) and so $X \pi Y$ will be complete, see [12, Ch.15, §64, Theorem 2]. Given $\varepsilon>0$ there exist $\left(x_{j}^{n}\right)_{j} \subset X$ and $\left(y_{j}^{n}\right)_{j} \subset Y$ such that $z_{n} \leq \sum_{j \geq 1}\left|x_{j}^{n} y_{j}^{n}\right|$ a.e. and $\sum_{j \geq 1}\left\|x_{j}^{n}\right\|_{X}\left\|y_{j}^{n}\right\|_{Y} \leq \pi\left(z_{n}\right)+\frac{\varepsilon}{2^{n}}$. So, $\sum_{n \geq 1} z_{n} \leq \sum_{n \geq 1} \sum_{j \geq 1}\left|x_{j}^{n} y_{j}^{n}\right|$ a.e. and

$$
\sum_{n \geq 1} \sum_{j \geq 1}\left\|x_{j}^{n}\right\|_{X}\left\|y_{j}^{n}\right\|_{Y} \leq \sum_{n \geq 1} \pi\left(z_{n}\right)+\varepsilon<\infty .
$$

Note that $z:=\sum_{n \geq 1} z_{n}<\infty$ a.e., since taking $h \in X^{Y^{\prime}}$ such that $h>0$ a.e., we have that

$$
\int|h z| d \mu \leq\|h\|_{X^{Y^{\prime}}} \sum_{n \geq 1} \sum_{j \geq 1}\left\|x_{j}^{n}\right\|_{X}\left\|y_{j}^{n}\right\|_{Y}<\infty
$$

which implies that $|h z|<\infty$ a.e. Then, $z \in X \pi Y$ and $\pi(z) \leq \sum_{n>1} \pi\left(z_{n}\right)$.

Therefore, it follows that $X \pi Y$ is a Banach function space. Moreover, given $A \in \Sigma$ with $\mu(A)>0$, since $X$ is saturated, there exists $B \in \Sigma$ such that $B \subset A, \mu(B)>0$ and $\chi_{B} \in X$. Since $Y$ is also saturated, there exists $C \in \Sigma$ such that $C \subset B, \mu(C)>0$ and $\chi_{C} \in Y$. Then, $\chi_{C}=\chi_{B} \cdot \chi_{C} \in X \pi Y$ and so $X \pi Y$ is saturated.

Suppose (a)-(b) holds. Claim (i) has been proved in (b) $\Rightarrow$ (a). The first equivalence in (ii) has been obtained in (a) $\Rightarrow(b)$. For the second equivalence, just note that $X \pi Y \equiv Y \pi X$.

The proof of the completeness of $X \pi Y$ in the previous proposition can also be obtained as a consequence of the fact that $X \pi Y$ is saturated. This can be found in [8], where these notions are developed in the general frame work of the function norms. The space defined in a similar way as $X \pi Y$ by taking finite sums has been independently studied in [11] obtaining similar results, although for the completeness the pointwise product $B_{X} \cdot B_{Y}$ of the unit balls of $X$ and $Y$ is required to be convex.

Remark 2.3. Suppose (a)-(b) in Proposition 2.2 holds. Then, if $\left(x_{i}\right) \subset X$ and $\left(y_{i}\right) \subset Y$ with $\sum_{i \geq 1}\left\|x_{i}\right\|_{X}\left\|y_{i}\right\|_{Y}<\infty$, we have that $\sum_{i \geq 1}\left|x_{i} y_{i}\right| \in X \pi Y$. Indeed, taking $h \in X^{Y^{\prime}}$ such that $h>0$ a.e., it follows that $\int h \sum_{i>1}\left|x_{i} y_{i}\right| d \mu<$ $\infty$ and so $\sum_{i \geq 1}\left|x_{i} y_{i}\right|<\infty$ a.e. As a consequence, every $z \in X \pi Y$ is actually an infinite sum of products of the type $x y$ with $x \in X$ and $y \in Y$. Indeed, if $\left(x_{i}\right) \subset X$ and $\left(y_{i}\right) \subset Y$ with $\sum_{i \geq 1}\left\|x_{i}\right\|_{X}\left\|y_{i}\right\|_{Y}<\infty$ and $|z| \leq$ $\sum_{i \geq 1}\left|x_{i} y_{i}\right|$ a.e., taking $v=\sum_{i \geq 1}\left|x_{i} y_{i}\right|$ and $\tilde{x}_{i}=\frac{z}{v} \chi_{\text {supp }(v)}\left|x_{i}\right| \in X$ (as 
$\left.\frac{z}{v} \chi_{\operatorname{supp}(v)} \in B_{L^{\infty}}\right)$, we have that $z=\sum_{i \geq 1} \tilde{x}_{i}\left|y_{i}\right|$ a.e. This fact may fail if $X^{Y^{\prime}}$ is not saturated. The series can be even divergent a.e. For instance, taking $x_{i}^{n}=\chi_{A_{i}^{n}} \in L^{1}$ and $y_{i}^{n}=\frac{1}{n} \chi_{A_{i}^{n}} \in L^{2}$ for $i=1, \ldots, n$ in Example 2, we have that

$$
\sum_{n \geq 1} \sum_{i=1}^{n}\left\|x_{i}^{n}\right\|_{L^{1}} \cdot\left\|y_{i}^{n}\right\|_{L^{2}}=\sum_{n \geq 1} \sum_{i=1}^{n} \frac{1}{n} \cdot \frac{1}{n}\left(\frac{1}{n}\right)^{\frac{1}{2}}=\sum_{n \geq 1} \frac{1}{n^{\frac{3}{2}}}<\infty,
$$

while $\sum_{n \geq 1} \sum_{i=1}^{n}\left|x_{i}^{n} y_{i}^{n}\right|=\sum_{n \geq 1} \frac{1}{n}=\infty$ a.e.

Example. Let us show some particular cases of $\pi$-product spaces.

(i) $X \pi L^{\infty} \equiv X$ even if $X$ is not saturated. This is direct from the definition of $\pi$-product space.

(ii) If $X$ is saturated, from a classical Lozanovskii's result ([7, Theorem 6]) it follows that $X \pi X^{\prime} \equiv L^{1}$.

(iii) Let $1 \leq p<\infty$. The $p$-power of a saturated Banach function space $X$ is the Banach function space given by $X^{p}=\left\{x \in L^{0}:|x|^{p} \in X\right\}$ with norm $\|x\|_{X^{p}}=\left\||x|^{p}\right\|_{X}^{1 / p}$ for $x \in X^{p}$, see [9, Proposition 1]. If $1 \leq r, q<\infty$ satisfy $1 / r=1 / p+1 / q$, from [9, Lemma 1$]$, it follows that $X^{p} \pi X^{q} \equiv X^{r}$. Moreover, if $Y$ is another Banach function space and $0<$ $\theta<1$ we obtain the Calderón-Lozanovskii interpolation space $X^{\theta} Y^{1-\theta}$ as the $\pi$-product space $X^{1 / \theta} \pi Y^{1 /(1-\theta)}$ (see [3] and [11, Section 2]).

We end this section with a result which will be useful along the paper.

Lemma 2.4. Assume that $X, Y$ and $Z$ are saturated Banach function spaces such that $X^{Z}$ and $Z^{Y}$ are saturated. Then, $X^{Z} \pi Z^{Y}$ is a saturated Banach function space and satisfies

$$
X^{Z} \pi Z^{Y} \hookrightarrow_{1} X^{Y}
$$

Proof. Let us see that $\left(X^{Z}\right)^{\left(Z^{Y}\right)^{\prime}}$ is saturated and so, by Proposition 2.2, we will have that $X^{Z} \pi Z^{Y}$ is a saturated Banach function space. Take $x \in X$ such that $x>0$ a.e. and $y^{\prime} \in Y^{\prime}$ such that $y^{\prime}>0$ a.e. Then $x y^{\prime}>0$ a.e. and for every $f \in X^{Z}$ and $g \in Z^{Y}$, as $Z^{Y} \hookrightarrow_{1} Z^{Y^{\prime \prime}} \equiv\left(Y^{\prime}\right)^{Z^{\prime}}$ (see for instance [2, $\S 2(3)$ and Lemma 3.1(a)] and Proposition 2.2(ii)), it follows

$$
\int\left|x y^{\prime} f g\right| d \mu \leq\|x f\|_{Z}\left\|y^{\prime} g\right\|_{Z^{\prime}}<\infty
$$

and so $x y^{\prime} \in\left(X^{Z}\right)^{\left(Z^{Y}\right)^{\prime}}$.

Given $z \in X^{Z} \pi Z^{Y}$, consider sequences $\left(f_{i}\right) \subset X^{Z}$ and $\left(g_{i}\right) \subset Z^{Y}$ satisfying that $\sum_{i \geq 1}\left\|f_{i}\right\|_{X^{Z}}\left\|g_{i}\right\|_{Z^{Y}}<\infty$ and $|z| \leq \sum_{i \geq 1}\left|f_{i} g_{i}\right|$ a.e. For every $x \in X$, we have that $|z x| \leq \sum_{i \geq 1}\left|f_{i} g_{i} x\right|$ a.e. with

$$
\sum_{i \geq 1}\left\|f_{i} g_{i} x\right\|_{Y} \leq \sum_{i \geq 1}\left\|f_{i} x\right\|_{Z}\left\|g_{i}\right\|_{Z^{Y}} \leq\|x\|_{X} \sum_{i \geq 1}\left\|f_{i}\right\|_{X^{Z}}\left\|g_{i}\right\|_{Z^{Y}}
$$

and so $z x \in Y$ with $\|z x\|_{Y} \leq\|x\|_{X} \cdot \pi(z)$. Hence, $z \in X^{Y}$ and satisfies $\|z\|_{X^{Y}} \leq \pi(z)$. 
Note that the hypothesis of Lemma 2.4 are satisfied for instance if $X$ is saturated and $X \subset Z \subset Y$ since in this case $L^{\infty}$ is contained in both $X^{Z}$ and $Z^{Y}$.

\section{The $d_{p, Z}$-product spaces}

Throughout this section, $X, Y$ and $Z$ will be saturated Banach function spaces such that $X^{Z}$ and $Z^{Y^{\prime}}$ are saturated. Then, by Lemma 2.4, we can consider the saturated Banach function space $X^{Z} \pi Z^{Y^{\prime}}$ which is contained in $X^{Y^{\prime}}$. In particular, $X^{Y^{\prime}}$ is saturated and so, by Proposition 2.2, we also can consider the saturated Banach function space $X \pi Y$.

Let $1 \leq p \leq \infty$. For any Banach space $E$ and $\left(e_{i}\right) \subset E$, we will denote

$$
\left\|\left(e_{i}\right)\right\|_{E, p}=\left(\sum_{i \geq 1}\left\|e_{i}\right\|_{E}^{p}\right)^{1 / p}
$$

if $p<\infty$ and for the case $p=\infty$,

$$
\left\|\left(e_{i}\right)\right\|_{E, \infty}=\sup _{i \geq 1}\left\|e_{i}\right\|_{E}
$$

Definition 3.1. The $d_{p, Z}$-product space $X d_{p, Z} Y$ is the space of functions $h \in$ $L^{0}$ such that $|h| \leq \sum_{i \geq 1}\left|x_{i} y_{i}\right|$ a.e. for some $\left(x_{i}\right) \subset X$ and $\left(y_{i}\right) \subset Y$ satisfying

$$
\left\|\left(y_{i}\right)\right\|_{Y, p^{\prime}} \cdot \sup _{f \in B_{X} Z}\left\|\left(f x_{i}\right)\right\|_{Z, p}<\infty
$$

where $1 \leq p^{\prime} \leq \infty$ is such that $1 / p+1 / p^{\prime}=1$. For $h \in X d_{p, Z} Y$, we denote

$$
d_{p, Z}(h)=\inf \left\{\left\|\left(y_{i}\right)\right\|_{Y, p^{\prime}} \cdot \sup _{f \in B_{X} Z}\left\|\left(f x_{i}\right)\right\|_{Z, p}\right\},
$$

where the infimum is taken over all $\left(x_{i}\right) \subset X,\left(y_{i}\right) \subset Y$ satisfying (3.1) such that $|h| \leq \sum_{i \geq 1}\left|x_{i} y_{i}\right|$ a.e.

Proposition 3.2. The space $X d_{p, Z} Y$ is a Banach function space with norm $d_{p, Z}$. Moreover,

$$
X d_{p, Z} Y \hookrightarrow_{1}\left(X^{Z} \pi Z^{Y^{\prime}}\right)^{\prime}
$$

Proof. Let $h \in X d_{p, Z} Y$ and take $\left(x_{i}\right) \subset X,\left(y_{i}\right) \subset Y$ satisfying (3.1) such that $|h| \leq \sum_{i \geq 1}\left|x_{i} y_{i}\right|$ a.e. Consider a function $\xi \in X^{Z} \pi Z^{Y^{\prime}}$ and $\left(f_{j}\right) \subset X^{Z}$, $\left(g_{j}\right) \subset Z^{Y^{\prime}} \equiv Y^{Z^{\prime}}$ with $|\xi| \leq \sum_{j \geq 1}\left|f_{j} g_{j}\right|$ a.e. and $\sum_{j \geq 1}\left\|f_{j}\right\|_{X^{Z}}\left\|g_{j}\right\|_{Y^{Z^{\prime}}}<\infty$. 
Then

$$
\begin{aligned}
\int|h \xi| d \mu & \leq \sum_{j \geq 1} \sum_{i \geq 1} \int\left|x_{i} y_{i} f_{j} g_{j}\right| d \mu \leq \sum_{j \geq 1} \sum_{i \geq 1}\left\|x_{i} f_{j}\right\|_{Z}\left\|y_{i} g_{j}\right\|_{Z^{\prime}} \\
& \leq \sum_{j \geq 1}\left\|g_{j}\right\|_{Y^{Z^{\prime}}} \sum_{i \geq 1}\left\|x_{i} f_{j}\right\|_{Z}\left\|y_{i}\right\|_{Y} \\
& \leq \sum_{j \geq 1}\left\|g_{j}\right\|_{Y^{Z^{\prime}}}\left\|\left(f_{j} x_{i}\right)_{i}\right\|_{Z, p}\left\|\left(y_{i}\right)\right\|_{Y, p^{\prime}} \\
& \leq\left(\sum_{j \geq 1}\left\|f_{j}\right\|_{X^{Z}}\left\|g_{j}\right\|_{Y^{Z^{\prime}}}\right) \cdot\left(\left\|\left(y_{i}\right)\right\|_{Y, p^{\prime}} \cdot \sup _{f \in B_{X^{Z}}}\left\|\left(f x_{i}\right)\right\|_{Z, p}\right) .
\end{aligned}
$$

So, $h \in\left(X^{Z} \pi Z^{Y^{\prime}}\right)^{\prime}$ and $\|h\|_{\left(X^{Z} \pi Z^{Y^{\prime}}\right)^{\prime}} \leq d_{p, Z}(h)$. In particular, $d_{p, Z}(h)=0$ implies $h=0$ a.e.

Note that if $h \in X d_{p, Z} Y$ and $\left(x_{i}\right) \subset X,\left(y_{i}\right) \subset Y$ are such that $|h| \leq$ $\sum_{i \geq 1}\left|x_{i} y_{i}\right|$ a.e. and satisfy $0<\left\|\left(y_{i}\right)\right\|_{Y, p^{\prime}} \cdot \sup _{f \in B_{X} Z}\left\|\left(f x_{i}\right)\right\|_{Z, p}<\infty$, then there exists $\left(\tilde{x}_{i}\right) \subset X$ and $\left(\tilde{y}_{i}\right) \subset Y$ such that $|h| \leq \sum_{i \geq 1}\left|\tilde{x}_{i} \tilde{y}_{i}\right|$ a.e.,

$$
\left\|\left(\tilde{y}_{i}\right)\right\|_{Y, p^{\prime}}=\left(\left\|\left(y_{i}\right)\right\|_{Y, p^{\prime}} \cdot \sup _{f \in B_{X} Z}\left\|\left(f x_{i}\right)\right\|_{Z, p}\right)^{1 / p^{\prime}}
$$

and

$$
\sup _{f \in B_{X} Z}\left\|\left(f \tilde{x}_{i}\right)\right\|_{Z, p}=\left(\left\|\left(y_{i}\right)\right\|_{Y, p^{\prime}} \cdot \sup _{f \in B_{X} Z}\left\|\left(f x_{i}\right)\right\|_{Z, p}\right)^{1 / p} .
$$

Indeed, the vectors

$$
\begin{aligned}
& \tilde{x}_{i}=\left\|\left(y_{i}\right)\right\|_{Y, p^{\prime}}^{1 / p} \cdot\left(\sup _{f \in B_{X} Z}\left\|\left(f x_{i}\right)\right\|_{Z, p}\right)^{-1 / p^{\prime}} \cdot x_{i}, \\
& \left.\tilde{y}_{i}=\left\|\left(y_{i}\right)\right\|_{Y, p^{\prime}}^{-1 / p} \cdot\left(\sup _{f \in B_{X} Z}\left\|\left(f x_{i}\right)\right\|_{Z, p}\right)\right)^{1 / p^{\prime}} \cdot y_{i}
\end{aligned}
$$

work.

Let $\left(h_{n}\right) \in X d_{p, Z} Y$ such that $\sum_{n \geq 1} d_{p, Z}\left(h_{n}\right)<\infty$. Let us prove that $h=\sum_{n \geq 1} h_{n} \in X d_{p, Z} Y$ with $d_{p, Z}(h) \leq \sum_{n \geq 1} d_{p, Z}\left(h_{n}\right)$ and so we will have that $d_{p, Z}$ satisfies the triangular inequality and $X d_{p, Z} Y$ has the RieszFischer property. Given $\varepsilon>0$, we can take $\left(x_{i}^{n}\right)_{i} \subset X,\left(y_{i}^{n}\right)_{i} \subset Y$ satisfying that $h_{n} \leq \sum_{i \geq 1}\left|x_{i}^{n} y_{i}^{n}\right|$ a.e. and

$$
\left\|\left(y_{i}^{n}\right)_{i}\right\|_{Y, p^{\prime}} \cdot \sup _{f \in B_{X} Z}\left\|\left(f x_{i}^{n}\right)_{i}\right\|_{Z, p} \leq d_{p, Z}\left(h_{n}\right)+\frac{\varepsilon}{2^{n}} .
$$

Note that $h \in L^{0}$, since taking $\xi \in X^{Z} \pi Z^{Y^{\prime}}$ such that $\xi>0$ a.e. and $\left(f_{j}\right)_{j} \in$ $X^{Z},\left(g_{j}\right)_{j} \in Z^{Y^{\prime}} \equiv Y^{Z^{\prime}}$ with $\sum_{j \geq 1}\left\|f_{j}\right\|_{X^{Z}}\left\|g_{j}\right\|_{Y^{Z^{\prime}}}<\infty$ and $\xi \leq \sum_{j \geq 1}\left|f_{j} g_{j}\right|$ 
a.e., denoting $\tilde{h}=\sum_{n \geq 1}\left|h_{n}\right|$ we have that

$$
\begin{aligned}
\int \tilde{h} \xi d \mu & \leq \sum_{j \geq 1} \sum_{n \geq 1} \sum_{i \geq 1} \int\left|x_{i}^{n} y_{i}^{n} f_{j} g_{j}\right| d \mu \leq \sum_{j \geq 1} \sum_{n \geq 1} \sum_{i \geq 1}\left\|x_{i}^{n} f_{j}\right\|_{Z}\left\|y_{i}^{n} g_{j}\right\|_{Z^{\prime}} \\
& \leq \sum_{j \geq 1}\left\|g_{j}\right\|_{Y^{\prime}} \sum_{n \geq 1} \sum_{i \geq 1}\left\|x_{i}^{n} f_{j}\right\|_{Z}\left\|y_{i}^{n}\right\|_{Y} \\
& \leq \sum_{j \geq 1}\left\|g_{j}\right\|_{Y^{Z^{\prime}}} \sum_{n \geq 1}\left(\left\|\left(y_{i}^{n}\right)_{i}\right\|_{Y, p^{\prime}} \cdot\left\|\left(f_{j} x_{i}^{n}\right)_{i}\right\|_{Z, p}\right) \\
& \leq\left(\sum_{j \geq 1}\left\|g_{j}\right\|_{Y^{Z^{\prime}}}\left\|f_{j}\right\|_{X^{Z}}\right) \cdot\left(\sum_{n \geq 1}\left(\left\|\left(y_{i}^{n}\right)_{i}\right\|_{Y, p^{\prime}} \cdot \sup _{f \in B_{X} Z}\left\|\left(f x_{i}^{n}\right)_{i}\right\|_{Z, p}\right)\right) \\
& \leq\left(\sum_{j \geq 1}\left\|g_{j}\right\|_{Y^{Z^{\prime}}}\left\|f_{j}\right\|_{X^{Z}}\right) \cdot\left(\varepsilon+\sum_{n \geq 1} d_{p, Z}\left(h_{n}\right)\right)<\infty
\end{aligned}
$$

and so $\tilde{h}<\infty$ a.e. We can assume that $\left\|\left(y_{i}^{n}\right)_{i}\right\|_{Y, p^{\prime}} \cdot \sup _{f \in B_{X} Z}\left\|\left(f x_{i}^{n}\right)_{i}\right\|_{Z, p}>0$ as in other case $d_{p, Z}\left(h_{n}\right)=0$ and so $h_{n}=0$ a.e. Consider $\left(\tilde{x}_{i}^{n}\right)_{i}$ and $\left(\tilde{y}_{i}^{n}\right)_{i}$ as above. Then, $|h| \leq \sum_{n \geq 1} \sum_{i \geq 1}\left|\tilde{x}_{i}^{n} \tilde{y}_{i}^{n}\right|$ a.e. and it can be checked that

$$
\left\|\left(\tilde{y}_{i}^{n}\right)_{n, i}\right\|_{Y, p^{\prime}} \cdot \sup _{f \in B_{X} Z}\left\|\left(f \tilde{x}_{i}^{n}\right)_{n, i}\right\|_{Z, p} \leq \sum_{n \geq 1}\left(\left\|\left(y_{i}^{n}\right)_{i}\right\|_{Y, p^{\prime}} \cdot \sup _{f \in B_{X} Z}\left\|\left(f x_{i}^{n}\right)_{i}\right\|_{Z, p}\right) .
$$

Thus, $h \in X d_{p, Z} Y$ and $d_{p, Z}(h) \leq \sum_{n \geq 1} d_{p, Z}\left(h_{n}\right)$.

The remaining conditions for $X \bar{d}_{p, Z} Y$ to be a Banach function space are clear and we have already shown in the beginning of the proof that $X d_{p, Z} Y \hookrightarrow_{1}\left(X^{Z} \pi Z^{Y^{\prime}}\right)^{\prime}$.

The norm $\pi$ of $X \pi Y$ can be described as follows. For every $z \in X \pi Y$,

$$
\pi(z)=\inf \left\{\left\|\left(x_{i}\right)\right\|_{X, p} \cdot\left\|\left(y_{i}\right)\right\|_{Y, p^{\prime}}\right\}
$$

where the infimum is taken over all sequences $\left(x_{i}\right) \subset X$ and $\left(y_{i}\right) \subset Y$ such that $|z| \leq \sum_{i \geq 1}\left|x_{i} y_{i}\right|$ a.e. and $\left\|\left(x_{i}\right)\right\|_{X, p} \cdot\left\|\left(y_{i}\right)\right\|_{Y, p^{\prime}}<\infty$. The proof of this fact is a routine computation after noting that if $\left(x_{i}\right) \subset X$ and $\left(y_{i}\right) \subset Y$ are such that $|z| \leq \sum_{i \geq 1}\left|x_{i} y_{i}\right|$ a.e., then for $\tilde{x}_{i}=\left(\left\|x_{i}\right\|_{X}\left\|y_{i}\right\|_{Y}\right)^{1 / p} \frac{x_{i}}{\left\|x_{i}\right\|_{X}} \in X$ and $\tilde{y}_{i}=\left(\left\|x_{i}\right\|_{X}\left\|y_{i}\right\|_{Y}\right)^{1 / p^{\prime}} \frac{y_{i}}{\left\|y_{i}\right\|_{Y}} \in Y$ we have that $\tilde{x}_{i} \tilde{y}_{i}=x_{i} y_{i}$ and $\left\|\left(\tilde{x}_{i}\right)\right\|_{X, p}$. $\left\|\left(\tilde{y}_{i}\right)\right\|_{Y, p^{\prime}}=\sum_{i \geq 1}\left\|x_{i}\right\|_{X}\left\|y_{i}\right\|_{Y}$. Since $X^{X} \equiv L^{\infty}$ (see [9, Theorem 1]) and

$$
\sup _{f \in B_{L} \infty}\left\|\left(f x_{i}\right)\right\|_{X, p}=\left\|\left(x_{i}\right)\right\|_{X, p},
$$

from (3.2), it follows that $X \pi Y \equiv X d_{p, X} Y$. Hence, the $\pi$-product spaces are

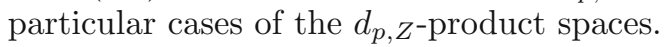

Proposition 3.3. The space $X d_{p, Z} Y$ is saturated and satisfies

$$
X \pi Y \hookrightarrow_{1} X d_{p, Z} Y
$$


Proof. Let $h \in X \pi Y$. By (3.2) there exist $\left(x_{i}\right) \subset X,\left(y_{i}\right) \subset Y$ such that $|h| \leq \sum_{i \geq 1}\left|x_{i} y_{i}\right|$ a.e. and $\left\|\left(x_{i}\right)\right\|_{X, p} \cdot\left\|\left(y_{i}\right)\right\|_{Y, p^{\prime}}<\infty$. Since

$$
\sup _{f \in B_{X} Z}\left\|\left(f x_{i}\right)\right\|_{Z, p} \leq\left\|\left(x_{i}\right)\right\|_{X, p}
$$

we have that $h \in X d_{p, z} Y$ and $d_{p, Z}(h) \leq \pi(z)$. Then, $X \pi Y \hookrightarrow_{1} X d_{p, Z} Y$ and in particular, $X d_{p, Z} Y$ is saturated.

\section{4. (p,Z)-summing multiplication operators}

Let us recall the definition given in (1.1). Given $X, Y, Z$ saturated Banach function spaces, a multiplication operator $g: X \rightarrow Y$ is $(p, Z)$-summing if there exists a constant $K>0$ such that for every $x_{1}, \ldots, x_{n} \in X$,

$$
\left(\sum_{i=1}^{n}\left\|g x_{i}\right\|_{Y}^{p}\right)^{1 / p} \leq K \sup _{f \in B_{X} Z}\left(\sum_{i=1}^{n}\left\|f x_{i}\right\|_{Z}^{p}\right)^{1 / p}
$$

if $1 \leq p<\infty$ and $\sup _{i=1, \ldots, n}\left\|g x_{i}\right\|_{Y} \leq K \sup _{f \in B_{X} Z}\left(\sup _{i=1, \ldots, n}\left\|f x_{i}\right\|_{Z}\right)$ if $p=\infty$.

Some relevant classes of multiplication operators between Banach function spaces can be obtained as particular $(p, Z)$-summing operators. Let us show some examples.

(I) $\boldsymbol{p}$-concave multiplication operators. Let $1 \leq p<\infty$. Recall that a linear operator $T: E \rightarrow F$, from a Banach lattice $E$ into a Banach space $F$, is $p$-concave if there exists $C>0$ such that for every $x_{1}, \ldots, x_{n} \in E$,

$$
\left(\sum_{i=1}^{n}\left\|T\left(x_{i}\right)\right\|_{F}^{p}\right)^{1 / p} \leq C\left\|\left(\sum_{i=1}^{n}\left|x_{i}\right|^{p}\right)^{1 / p}\right\|_{E} .
$$

Every $\left(p, L^{p}\right)$-summing multiplication operator $g: X \rightarrow Y$ is $p$-concave. Indeed, noting that $X \hookrightarrow_{1} X^{L^{p} L^{p}}$ (see for instance [2, $\left.\S 2(3)\right]$ ), we have that there exists $K>0$ such that for every $x_{1}, \ldots, x_{n} \in X$,

$$
\begin{aligned}
\left(\sum_{i=1}^{n}\left\|g x_{i}\right\|_{Y}^{p}\right)^{1 / p} & \leq K \sup _{f \in B_{X} L^{p}}\left(\sum_{i=1}^{n}\left\|f x_{i}\right\|_{L^{p}}^{p}\right)^{1 / p} \\
& =K \sup _{f \in B_{X} L^{p}}\left\|f\left(\sum_{i=1}^{n}\left|x_{i}\right|^{p}\right)^{1 / p}\right\|_{L^{p}} \\
& =K\left\|\left(\sum_{i=1}^{n}\left|x_{i}\right|^{p}\right)^{1 / p}\right\|_{X^{L^{p} L^{p}}} \\
& \leq K\left\|\left(\sum_{i=1}^{n}\left|x_{i}\right|^{p}\right)^{1 / p}\right\|_{X} .
\end{aligned}
$$


Recall that a Banach lattice $E$ is $p$-convex with constant $K$ if for all $x_{1}, \ldots, x_{n} \in E$,

$$
\left\|\left(\sum_{i=1}^{n}\left|x_{i}\right|^{p}\right)^{1 / p}\right\|_{E} \leq K\left(\sum_{i=1}^{n}\left\|x_{i}\right\|_{E}^{p}\right)^{1 / p} .
$$

If the contrary inequality holds, then $E$ is called $p$-concave. In the case when $X$ is $p$-convex with constant 1 and order semi-continuous, the inequality (4.1) is an equality (see [2, Proposition 5.3(ii)]) and so the class of the $p$-concave multiplication operators from $X$ into $Y$ coincides with the class of the $\left(p, L^{p}\right)$-summing ones.

(II) Positive $\boldsymbol{p}$-summing multiplication operators. Let $1 \leq p<\infty$. An operator $T: E \rightarrow F$, with $E$ a Banach lattice and $F$ a Banach space, is positive $p$-summing if there exists $K>0$ such that for every $x_{1}, \ldots, x_{n} \in E$,

$$
\left(\sum_{i=1}^{n}\left\|T\left(\left|x_{i}\right|\right)\right\|^{p}\right)^{1 / p} \leq K \sup _{x^{*} \in B_{E^{*}}}\left(\sum_{i=1}^{n}\left|\left\langle x^{*},\left|x_{i}\right|\right\rangle\right|^{p}\right)^{1 / p},
$$

where $E^{*}$ is the topological dual of $E$, see [1]. Every $\left(p, L^{1}\right)$-summing multiplication operator $g: X \rightarrow Y$ is positive $p$-summing. Indeed, noting that each $f \in X^{\prime}$ can be identified with an element of the dual space $X^{*}$ via $\langle f, x\rangle=\int f x d \mu$ for all $x \in X$ with $\|f\|_{X^{\prime}}=\|f\|_{X^{*}}$, we have that there exists $K>0$ such that for every $x_{1}, \ldots, x_{n} \in X$,

$$
\begin{aligned}
\left(\sum_{i=1}^{n}\left\|g\left|x_{i}\right|\right\|_{Y}^{p}\right)^{1 / p} & =\left(\sum_{i=1}^{n}\left\|g x_{i}\right\|_{Y}^{p}\right)^{1 / p} \\
& \leq K \sup _{f \in B_{X^{L^{1}}}}\left(\sum_{i=1}^{n}\left\|f x_{i}\right\|_{L^{1}}^{p}\right)^{1 / p} \\
& =K \sup _{f \in B_{X^{\prime}}}\left(\sum_{i=1}^{n}\left\langle|f|,\left|x_{i}\right|\right\rangle^{p}\right)^{1 / p} \\
& \leq K \sup _{x^{*} \in B_{X^{*}}}\left(\sum_{i=1}^{n}\left|\left\langle x^{*},\left|x_{i}\right|\right\rangle\right|^{p}\right)^{1 / p} .
\end{aligned}
$$

In the case when $X$ is order continuous, the Köthe dual $X^{\prime}$ can be identified with the whole space $X^{*}$ (see for instance [6, p. 29]) and then the inequality (4.2) is just an equality, so the class of the positive $p$-summing multiplication operators from $X$ into $Y$ coincides with the class of the $\left(p, L^{1}\right)$-summing ones. The analogous result holds for $p=\infty$.

From now and on $X, Y$ and $Z$ will be saturated Banach function spaces such that $X^{Z}$ and $Z^{Y}$ are saturated. In this case, by Lemma 2.4, we have that $X^{Z} \pi Z^{Y}$ is a saturated Banach function space. Moreover, since $Z^{Y^{\prime \prime}}$ is also saturated (as it contains $Z^{Y}$ ), we can consider the space $X d_{p, Z} Y^{\prime}$. Let us show now our main result which gives a characterization of the space of all $(p, Z)$-summing multiplication operators from $X$ into $Y$, whenever $Y$ is order semi-continuous. Note that, adopting the notation given in Section 3, 
a function $g \in X^{Y}$ is $(p, Z)$-summing if there exists a constant $K>0$ such that for every $x_{1}, \ldots, x_{n} \in X$,

$$
\left\|\left(g x_{i}\right)\right\|_{Y, p} \leq K \sup _{f \in B_{X} Z}\left\|\left(f x_{i}\right)\right\|_{Z, p}
$$

In this case, the inequality also holds for infinite sequences.

Theorem 4.1. Assume that $Y$ is order semi-continuous and let $g: X \rightarrow Y$ be a multiplication operator. Then, $g$ is $(p, Z)$-summing if and only if $g \in$ $\left(X d_{p, Z} Y^{\prime}\right)^{\prime}$.

Proof. Suppose that $g$ is $(p, Z)$-summing. Given $h \in X d_{p, Z} Y^{\prime}$ and $\left(x_{i}\right) \subset X$, $\left(y_{i}^{\prime}\right) \subset Y^{\prime}$ satisfying $(3.1)$ for $Y^{\prime}$ instead of $Y$ and such that $|h| \leq \sum_{i \geq 1}\left|x_{i} y_{i}^{\prime}\right|$ a.e., we have that

$$
\begin{aligned}
\int|g h| d \mu & \leq \sum_{i \geq 1} \int\left|g x_{i} y_{i}^{\prime}\right| d \mu \\
& \leq \sum_{i \geq 1}\left\|g x_{i}\right\|_{Y}\left\|y_{i}^{\prime}\right\|_{Y^{\prime}} \\
& \leq\left\|\left(g x_{i}\right)\right\|_{Y, p} \cdot\left\|\left(y_{i}^{\prime}\right)\right\|_{Y^{\prime}, p^{\prime}} \\
& \leq K\left\|\left(y_{i}^{\prime}\right)\right\|_{Y^{\prime}, p^{\prime}} \cdot \sup _{f \in B_{X} Z}\left\|\left(f x_{i}\right)\right\|_{Z, p}<\infty
\end{aligned}
$$

and so $g \in\left(X d_{p, Z} Y^{\prime}\right)^{\prime}$.

Let us prove the converse. Suppose that $g \in\left(X d_{p, Z} Y^{\prime}\right)^{\prime}$ and let $x_{1}, \ldots, x_{n} \in X$. Suppose first that $1 \leq p<\infty$. Given $\varepsilon>0$, since $\|y\|_{Y}=$ $\|y\|_{Y^{\prime \prime}}$ for all $y \in Y$ as $Y$ is order semi-continuous (see for instance [2, p. 4,5]), there exists $y_{i}^{\prime} \in B_{Y^{\prime}}$ such that $\left\|g x_{i}\right\|_{Y} \leq \varepsilon c+\int\left|g x_{i} y_{i}^{\prime}\right| d \mu$, where $c=\left(\sum_{i=1}^{n}\left\|g x_{i}\right\|_{Y}^{p}\right)^{1 / p^{\prime}} /\left(\sum_{i=1}^{n}\left\|g x_{i}\right\|_{Y}^{p-1}\right)$. Then, denoting $\tilde{y}_{i}^{\prime}=\left\|g x_{i}\right\|_{Y}^{p-1} y_{i}^{\prime}$, we have that

$$
\begin{aligned}
\sum_{i=1}^{n}\left\|g x_{i}\right\|_{Y}^{p} & =\sum_{i=1}^{n}\left\|g x_{i}\right\|_{Y}^{p-1}\left\|g x_{i}\right\|_{Y} \\
& \leq \sum_{i=1}^{n}\left\|g x_{i}\right\|_{Y}^{p-1}\left(\varepsilon c+\int\left|g x_{i} y_{i}^{\prime}\right| d \mu\right) \\
& =\varepsilon\left(\sum_{i=1}^{n}\left\|g x_{i}\right\|_{Y}^{p}\right)^{1 / p^{\prime}}+\int|g| \sum_{i=1}^{n}\left|x_{i} \tilde{y}_{i}^{\prime}\right| d \mu \\
& \leq \varepsilon\left(\sum_{i=1}^{n}\left\|g x_{i}\right\|_{Y}^{p}\right)^{1 / p^{\prime}}+\|g\|_{\left(X d_{p, Z} Y^{\prime}\right)^{\prime}} d_{p, Z}\left(\sum_{i=1}^{n}\left|x_{i} \tilde{y}_{i}^{\prime}\right|\right)
\end{aligned}
$$


Noting that

$$
\begin{aligned}
d_{p, Z}\left(\sum_{i=1}^{n}\left|x_{i} \tilde{y}_{i}^{\prime}\right|\right) & \leq\left(\sum_{i=1}^{n}\left\|\tilde{y}_{i}^{\prime}\right\|_{Y^{\prime}}^{p^{\prime}}\right)^{1 / p^{\prime}} \cdot \sup _{f \in B_{X} Z}\left(\sum_{i=1}^{n}\left\|f x_{i}\right\|_{Z}^{p}\right)^{1 / p} \\
& \leq\left(\sum_{i=1}^{n}\left\|g x_{i}\right\|_{Y}^{(p-1) p^{\prime}}\right)^{1 / p^{\prime}} \cdot \sup _{f \in B_{X} Z}\left(\sum_{i=1}^{n}\left\|f x_{i}\right\|_{Z}^{p}\right)^{1 / p},
\end{aligned}
$$

we obtain

$\sum_{i=1}^{n}\left\|g x_{i}\right\|_{Y}^{p} \leq\left(\sum_{i=1}^{n}\left\|g x_{i}\right\|_{Y}^{p}\right)^{1 / p^{\prime}} \cdot\left(\varepsilon+\|g\|_{\left(X d_{p, Z} Y^{\prime}\right)^{\prime}} \sup _{f \in B_{X} Z}\left(\sum_{i=1}^{n}\left\|f x_{i}\right\|_{Z}^{p}\right)^{1 / p}\right)$

and so

$$
\left(\sum_{i=1}^{n}\left\|g x_{i}\right\|_{Y}^{p}\right)^{1 / p} \leq \varepsilon+\|g\|_{\left(X d_{p, Z} Y^{\prime}\right)^{\prime}} \sup _{f \in B_{X} Z}\left(\sum_{i=1}^{n}\left\|f x_{i}\right\|_{Z}^{p}\right)^{1 / p} .
$$

Since $\varepsilon$ is arbitrary, $g$ is $(p, Z)$-summing. For the case $p=\infty$, given $\varepsilon>0$, there exists $y_{i}^{\prime} \in B_{Y^{\prime}}$ such that $\left\|g x_{i}\right\|_{Y} \leq \varepsilon+\int\left|g x_{i} y_{i}^{\prime}\right| d \mu$, and then

$$
\begin{aligned}
\sup _{i=1, \ldots, n}\left\|g x_{i}\right\|_{Y} & \leq \varepsilon+\sup _{i=1, \ldots, n} \int\left|g x_{i} y_{i}^{\prime}\right| d \mu \\
& \leq \varepsilon+\|g\|_{\left(X d_{\infty, Z} Y^{\prime}\right)^{\prime}} \sup _{i=1, \ldots n} d_{\infty, Z}\left(x_{i} y_{i}^{\prime}\right) \\
& \leq \varepsilon+\|g\|_{\left(X d_{\infty, Z} Y^{\prime}\right)^{\prime}} \sup _{i=1, \ldots n}\left(\left\|y_{i}^{\prime}\right\|_{Y} \cdot \sup _{f \in B_{X} Z}\left\|f x_{i}\right\|_{Z}\right) \\
& \leq \varepsilon+\|g\|_{\left(X d_{\infty, Z} Y^{\prime}\right)^{\prime}} \sup _{f \in B_{X} Z}\left(\sup _{i=1, \ldots n}\left\|f x_{i}\right\|_{Z}\right)
\end{aligned}
$$

so $g$ is $(\infty, Z)$-summing.

Note that under conditions of Theorem 4.1, from the proof it follows that $\|g\|_{\left(X d_{p, Z} Y^{\prime}\right)^{\prime}}$ is the smallest constant $K$ satisfying the inequality of the definition of $(p, Z)$-summing.

By Proposition 3.2 and Proposition 3.3,

$$
X \pi Y^{\prime} \hookrightarrow_{1} X d_{p, Z} Y^{\prime} \hookrightarrow_{1}\left(X^{Z} \pi Z^{Y^{\prime \prime}}\right)^{\prime}
$$

Then, $\left(X^{Z} \pi Z^{Y^{\prime \prime}}\right)^{\prime \prime} \hookrightarrow_{1}\left(X d_{p, Z} Y^{\prime}\right)^{\prime} \hookrightarrow_{1}\left(X \pi Y^{\prime}\right)^{\prime}$ (see for instance [2, Lemma 3.1(b)]) and since $X^{Z} \pi Z^{Y} \hookrightarrow_{1} X^{Z} \pi Z^{Y^{\prime \prime}} \hookrightarrow_{1}\left(X^{Z} \pi Z^{Y^{\prime \prime}}\right)^{\prime \prime}$ and $\left(X \pi Y^{\prime}\right)^{\prime} \equiv X^{Y^{\prime \prime}}$ (see Proposition 2.2(ii)), we have that

$$
X^{Z} \pi Z^{Y} \hookrightarrow_{1}\left(X d_{p, Z} Y^{\prime}\right)^{\prime} \hookrightarrow_{1} X^{Y^{\prime \prime}}
$$

On the other hand, from Lemma 2.4, it follows that

$$
X^{Z} \pi Z^{Y} \hookrightarrow_{1} X^{Y} \hookrightarrow_{1} X^{Y^{\prime \prime}}
$$

Rewriting Theorem 4.1, whenever $Y$ is order semi-continuous, we get

$$
\left\{g \in X^{Y}: g \text { is }(p, Z) \text {-summing }\right\}=X^{Y} \cap\left(X d_{p, Z} Y^{\prime}\right)^{\prime} .
$$


In the case when $Y$ has the Fatou property (i.e. $Y \equiv Y^{\prime \prime}$ ), from (4.3), it follows that the space of $(p, Z)$-summing multiplication operators from $X$ into $Y$ is just $\left(X d_{p, Z} Y^{\prime}\right)^{\prime}$, in particular, it has the Fatou property as it coincides with the Köthe dual of a Banach function space.

From (4.3), (4.4) and Theorem 4.1, we obtain that under the assumption of order semi-continuity for $Y$, if $g \in X^{Z} \pi Z^{Y}$ then $g$ is a $(p, Z)$-summing multiplication operator from $X$ into $Y$. A direct computation proves that this holds also without any assumption on $Y$. In particular, if a multiplication operator $g: X \rightarrow Y$ factorizes through $Z$ via two multiplication operators (i.e. $g=f h$ for some $f \in X^{Z}$ and $\left.h \in Z^{Y}\right)$, then $g$ is $(p, Z)$-summing. Let us show a useful consequence of $(4.3),(4.4)$ and Theorem 4.1 .

Corollary 4.2. Let $Y$ a Banach function space with the Fatou property and suppose that $X^{Z} \pi Z^{Y}=X^{Y}$. Then

$$
\left\{g \in X^{Y}: g \text { is }(p, Z) \text {-summing }\right\}=\left(X d_{p, Z} Y^{\prime}\right)^{\prime}=X^{Y} .
$$

The Fatou property for $Y$ is necessary in the result above. An easy counterexample can be given if it is not satisfied. Take $X=\ell^{\infty}, Y=c_{0}$ and $Z=\ell^{\infty}$. Then $X^{Y}=c_{0}$ and $X^{Z} \pi Z^{Y}=\ell^{\infty} \pi c_{0}=c_{0}=X^{Y}$. However, $\ell^{1}=\ell^{\infty} \pi \ell^{1}=\ell^{\infty} d_{p, \ell \infty} \ell^{1}$ (see the comments before Proposition 3.3), and then $\left(X d_{p, Z} Y^{\prime}\right)^{\prime}=\left(\ell^{\infty} d_{p, \ell \infty} \ell^{1}\right)^{\prime}=\ell^{\infty}$.

Corollary 4.2 provides conditions guaranteeing that the space consisting of all $(p, Z)$-summing multiplication operators from $X$ into $Y$ coincides with the whole space $X^{Y}$. This is not a general fact, as the following example shows.

Example. Consider $1<p<q<r<\infty$ and the spaces $X=\ell^{p}, Y=\ell^{q}$ and $Z=\ell^{r}$. Note that, since $X^{Z} \equiv X^{Y} \equiv \ell^{\infty}$ and $Z^{Y} \equiv \ell^{s}$ for $1 / s=1 / q-1 / r$ (see $\left[9\right.$, Theorem 2 and Proposition 3]), in this case $X^{Z} \pi Z^{Y} \equiv \ell^{\infty} \pi \ell^{s} \equiv \ell^{s} \varsubsetneqq X^{Y}$. The space of $\left(t, \ell^{r}\right)$-summing multiplication operators from $\ell^{p}$ into $\ell^{q}$ is just the space of sequences $g \in \ell^{\infty}$ satisfying

$$
\left\|\left(g x_{i}\right)\right\|_{\ell^{q}, t} \leq K \sup _{f \in B_{\ell} \infty}\left\|\left(f x_{i}\right)\right\|_{\ell^{r}, t}=K\left\|\left(x_{i}\right)\right\|_{\ell^{r}, t}
$$

for some constant $K>0$ and for every $x_{1}, \ldots, x_{n} \in \ell^{p}$. Clearly, there exist elements of $\ell^{\infty}$ which do not satisfy the above inequality (e.g. $\left.g=(1,1,1, \ldots)\right)$. Note that, since $X^{Z} \pi Z^{Y} \equiv \ell^{s}$, every $g \in \ell^{s}$ is $\left(t, \ell^{r}\right)$-summing for all $1 \leq t \leq \infty$.

Other conditions different from those in Corollary 4.2 under which every multiplication operators from $X$ into $Y$ is $(p, Z)$-summing are presented in the following result.

Proposition 4.3. Assume $Y$ is p-concave, $Z$ is $p$-convex and $X \hookrightarrow_{i} X^{Z Z}$. Then, every $g \in X^{Y}$ is $(p, Z)$-summing. 
Proof. Let $g \in X^{Y}$. Given $x_{1}, \ldots, x_{n} \in X$, we have that

$$
\begin{aligned}
\left(\sum_{i=1}^{n}\left\|g x_{i}\right\|_{Y}^{p}\right)^{1 / p} & \leq K_{1}\left\|\left(\sum_{i=1}^{n}\left|g x_{i}\right|^{p}\right)^{1 / p}\right\|_{Y} \\
& \leq K_{1}\|g\|_{X^{Y}}\left\|\left(\sum_{i=1}^{n}\left|x_{i}\right|^{p}\right)^{1 / p}\right\|_{X} \\
& =K_{1}\|g\|_{X^{Y}} \sup _{f \in B_{X Z}}\left\|\left(\sum_{i=1}^{n}\left|f x_{i}\right|^{p}\right)^{1 / p}\right\|_{Z} \\
& \leq K_{1} K_{2}\|g\|_{X^{Y}} \sup _{f \in B_{X Z}}\left(\sum_{i=1}^{n}\left\|f x_{i}\right\|_{Z}^{p}\right)^{1 / p},
\end{aligned}
$$

where $K_{1}$ is the $p$-concavity constant of $Y, K_{2}$ is the $p$-convexity constant of $Z$ and for the equality we have used that $\|x\|_{X}=\|x\|_{X Z Z}$ for all $x \in X$.

Actually in Proposition $4.3 X$ and $X^{Z}$ being saturated are enough instead of the saturation conditions required before Theorem 4.1. Conditions under which $X \hookrightarrow_{i} X^{Z Z}$ are studied in [2]. Finally, note that every multiplication operator from $X$ into $Y$ is $(p, X)$-summing and $(p, Y)$-summing.

\section{Applications}

Let us finish the paper by applying several important factorization theorems for multiplication operators to the results obtained for the $d_{p, Z \text {-product }}$ spaces. Summability properties of these operators are obtained in a straightforward way from Corollary 4.2 .

\subsection{Reisner's theorem}

Let $Z$ be a Banach function space satisfying that $L_{F}^{\infty} \subset Z \subset L_{l o c}^{1}$, where $L_{F}^{\infty}$ denotes the space of functions in $L^{\infty}$ with support having finite measure and $L_{l o c}^{1}$ denotes the space of locally integrable functions. Given $1 \leq p<q \leq \infty$, consider $r$ defined by $1 / r=1 / p-1 / q$. If $Z$ is $p$-convex with constant $K_{1}$ and $q$-concave with constant $K_{2}$, then for every $\varepsilon>0$ and $g \in\left(L^{q}\right)^{L^{p}} \equiv L^{r}$ (see [9, Proposition 3]), the multiplication operator $g: L^{q} \rightarrow L^{p}$ has a factorization as

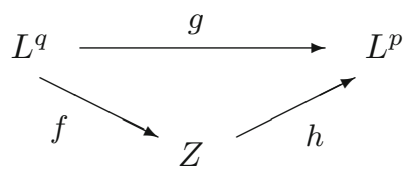

(i.e. $g=f h$ ) where $f \in\left(L^{q}\right)^{Z}$ and $h \in Z^{L^{p}}$ are such that

$$
\|f\|_{\left(L^{q}\right)^{Z}} \cdot\|h\|_{Z^{L^{p}}} \leq(1+\varepsilon) K_{1} K_{2}\|g\|_{\left(L^{q}\right)^{L^{p}}}
$$

(see [10, Theorem 1]) and so $g \in\left(L^{q}\right)^{Z} \pi Z^{L^{p}}$ with $\pi(g) \leq K_{1} K_{2}\|g\|_{\left(L^{q}\right)^{L^{p}}}$. Hence, $\left(L^{q}\right)^{L^{p}} \hookrightarrow_{K_{1} K_{2}}\left(L^{q}\right)^{Z} \pi Z^{L^{p}}$. Note that $Z^{L^{p}}$ and $\left(L^{q}\right)^{Z}$ are saturated, see the comments in [2] after Proposition 5.3 and Theorem 5.4. Then, by 
Lemma 2.4, we have $\left(L^{q}\right)^{Z} \pi Z^{L^{p}} \hookrightarrow_{1}\left(L^{q}\right)^{L^{p}}$. Hence, $\left(L^{q}\right)^{Z} \pi Z^{L^{p}}=\left(L^{q}\right)^{L^{p}}$ (with equal norms if $K_{1} K_{2} \leq 1$ ). Therefore, from Corollary 4.2, we obtain the following result.

Proposition 5.1. Let $1 \leq p<q \leq \infty$ and $r$ such that $1 / r=1 / p-1 / q$. If $Z$ is a p-convex and $q$-concave Banach function space such that $L_{F}^{\infty} \subset Z \subset L_{\text {loc }}^{1}$, then the space of $(s, Z)$-summing multiplication operators from $L^{q}$ into $L^{p}$ (for any $1 \leq s \leq \infty$ ) coincides with the whole space $L^{r}$, which also coincides with $\left(L^{q} d_{s, Z} L^{p^{\prime}}\right)^{\prime}$.

Note that under conditions of Proposition 5.1, the norm $\pi$ of $\left(L^{q}\right)^{Y} \pi Y^{L^{p}}$ is equivalent to $\tilde{\pi}$, defined on $g \in\left(L^{q}\right)^{Y} \pi Y^{L^{p}}$ as

$$
\tilde{\pi}(g)=\inf \left\{\|f\|_{\left(L^{q}\right)^{Y}}\|h\|_{Y^{L^{p}}}: g=f h \text { with } f \in\left(L^{q}\right)^{Y} \text { and } h \in Y^{L^{p}}\right\} \text {. }
$$

The norms $\pi$ and $\tilde{\pi}$ coincide whenever $K_{1} K_{2} \leq 1$.

\subsection{Maurey-Rosenthal's theorem}

Let $1 \leq p<\infty$. Recall that a linear operator $T: E \rightarrow F$, from a Banach space $E$ into a Banach lattice $F$, is $p$-convex if there exists $C>0$ such that for every $x_{1}, \ldots, x_{n} \in E$,

$$
\left\|\left(\sum_{i=1}^{n}\left|T\left(x_{i}\right)\right|^{p}\right)^{1 / p}\right\|_{F} \leq C\left(\sum_{i=1}^{n}\left\|x_{i}\right\|_{E}^{p}\right)^{1 / p} .
$$

Consider a saturated Banach function space $Y$ being order semi-continuous and $p$-concave with constant $K_{1}$. If $T: E \rightarrow Y$ is $p$-convex with constant $K_{2}$, then there exists a function $0 \leq h \in\left(L^{p}\right)^{Y}$ and an operator $R: E \rightarrow L^{p}$ such that $T$ factorizes as

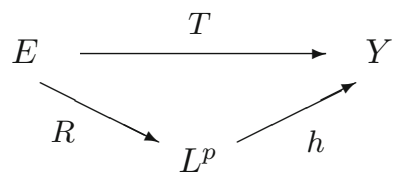

and $\|h\|_{\left(L^{p}\right)^{Y}}\|R\|_{E \rightarrow L^{p}} \leq K_{1} K_{2}$ (see [4, Corollary 2]). If a saturated Banach function space $X$ is $p$-convex with constant $K$, it is direct to check that every multiplication operator $g: X \rightarrow Y$ is $p$-convex with constant $K\|g\|_{X^{Y}}$. Then, there exists $0 \leq h \in\left(L^{p}\right)^{Y}$ and an operator $R: X \rightarrow L^{p}$ such that $g x=h R(x)$ for all $x \in X$ and $\|h\|_{\left(L^{p}\right)^{Y}}\|R\|_{X \rightarrow L^{p}} \leq K_{1} K\|g\|_{X^{Y}}$. Taking $f:=\frac{g}{h} \chi_{[h>0]}$, we have that $f \in X^{L^{p}}$ (as $|f x| \leq|R(x)|$ for all $x \in X$ ) and $g=f h$ (as $g x=h R(x)$ with $0<x \in X$ implies $g(\omega)=0$ whenever $h(\omega)=0)$. Then, $g \in X^{L^{p}} \pi\left(L^{p}\right)^{Y}$ with $\pi(g) \leq K_{1} K\|g\|_{X^{Y}}$. Hence, $X^{Y} \hookrightarrow K_{1} K X^{L^{p}} \pi\left(L^{p}\right)^{Y}$. Suppose that $\left(L^{p}\right)^{Y}$ is saturated. For instance, this is the case when $L_{F}^{\infty} \subset$ $Y \subset L_{l o c}^{1}$, as $Y$ is $p$-concave. Since $X^{L^{p}}$ is saturated (as $X$ is $p$-convex), by Lemma 2.4 we have that $X^{L^{p}} \pi\left(L^{p}\right)^{Y} \hookrightarrow_{1} X^{Y}$. So, $X^{L^{p}} \pi\left(L^{p}\right)^{Y}=X^{Y}$ (with equal norms if $K_{1} K \leq 1$ ). Therefore, from Corollary 4.2, we obtain the following result. 
Proposition 5.2. Let $1 \leq p<\infty$. Given two saturated Banach function spaces $X$ and $Y$ such that $X$ is $p$-convex and $Y$ is p-concave, has the Fatou property and satisfies that $\left(L^{p}\right)^{Y}$ is saturated, then the space of $\left(s, L^{p}\right)$-summing multiplication operators from $X$ into $Y$ (for any $1 \leq s \leq \infty$ ) coincides with the whole space $X^{Y}$, which also coincides with $\left(X d_{s, L^{p}} Y^{\prime}\right)^{\prime}$.

Note that under conditions of Proposition 5.2, the norm $\pi$ of $X^{L^{p}} \pi\left(L^{p}\right)^{Y}$ is equivalent to $\tilde{\pi}$ defined on $X^{L^{p}} \pi\left(L^{p}\right)^{Y}$ in a similar way as (5.1). If $K_{1} K \leq 1$, the norms $\pi$ and $\tilde{\pi}$ coincide.

\section{Acknowledgment}

The authors thank the referee for providing the reference to the paper by Luxemburg and Zaanen quoted after the proof of Proposition 2.2.

\section{References}

[1] O. Blasco, Positive p-summing operators on $L_{p}$-spaces, Proc. Amer. Math. Soc. 100 (1987), 275-280.

[2] J. M. Calabuig, O. Delgado and E. A. Sánchez Pérez, Generalized perfect spaces, Indag. Math. 19 (2008), 359-378.

[3] A. P. Calderón, Intermediate spaces and interpolation, the complex method, Studia Math. 24 (1964), 113-190.

[4] A. Defant, Variants of the Maurey-Rosenthal Theorem for quasi-Köthe function spaces, Positivity 5 (2001), 153-175.

[5] A. Defant and K. Floret, Tensor norms and operator ideals, North Holland Math. Studies, Amsterdam, 1993.

[6] J. Lindenstrauss and L. Tzafriri, Classical Banach Spaces II, Springer-Verlag, Berlin, 1979.

[7] G. Ya. Lozanovskii, On some Banach lattices, Siberian Math. J. 10 (1969), 419-430.

[8] W. A. J. Luxemburg and A. C. Zaanen, Notes on Banach function spaces, Note III, Nederl. Akad. Wet., Proc., Ser. A 66 (1963), 239-250.

[9] L. Maligranda and L. E. Persson, Generalized duality of some Banach function spaces, Indag. Math. 51 (1989), 323-338.

[10] S. Reisner, A factorization theorem in Banach lattices and its application to Lorentz spaces, Annales de l'institut Fourier 31 (1981), 239-255.

[11] A. R. Schep, Products and factors of Banach function spaces, preprint.

[12] A. C. Zaanen, Integration, 2nd rev. ed. North Holland, Amsterdam; Interscience, New York, 1967. 